\title{
Journal of Vaseular Research
}

Piyachat Chansela, Bangkok, Thailand Christian Aalkjaer, Aarhus, Denmark Kohsuke Adachi, Kochi, Japan Blerina Ahmetaj-Shala, London, UK Francisco Alcaín, Ciudad Real, Spain Yvonne Alexander, Manchester, UK Javier Gomez Ambrosi, Pamplona, Spain Kewal Asosingh, Cleveland, $\mathrm{OH}$, USA Arif Aydın, Konya, Turkey Ildiko Bácskay, Debrecen, Hungary Xue Bai, Chapel Hill, NC, USA Pedro Baptista, Zaragoza, Spain Fellype Barreto, Curitiba, Brazil Manuela Bartoli, Augusta, GA, USA Friedhelm Beyersdorf, Freiburg, Germany Alex Bobik, Melbourne, VIC, Australia Francesca Boccafoschi, Novara, Italy Francesca Bonomini, Brescia, Italy Malika Bouchenak, Oran, Algeria Eliete Bouskela, Rio de Janeiro, Brazil Doug Bowles, Columbia, MO, USA Luke P. Brewster, Atlanta, GA, USA Sergey Brodsky, Columbus, OH, USA Mariarosaria Bucci, Naples, Italy Hendrik Buikema, Groningen,

The Netherlands Albert Busch, Würzburg, Germany Ilaria Campesi, Sassari, Italy Federica Cerini, Colleferro, Italy Nabil Chakfe, Strasbourg, France Lihui Cheng, Shanghai, China Peter Claus, Hannover, Germany Jennifer E. Cole, Oxford, UK Gabor Csanyi, Augusta, GA, USA Pedro Cuevas, Madrid, Spain Green J. Daniel, Perth, WA, Australia Sergio De Marchi, Verona, Italy Jo De Mey, Odense, Denmark Vivian de Waard, Amsterdam, The Netherlands Cor de Wit, Lübeck, Germany Elisabeth Deindl, Munich, Germany Dominic Del Re, Rutgers, NJ, USA Chantal Dessy, Woluwe-Saint-Lambert, Belgium

The Editors would like to thank the following individuals for their expert assistance in acting as reviewers for the Journal of Vascular Research in the period of December 2016 to November 2017. Together with our authors and readers we appreciate the time they have freely given.

Kim Dora, Oxford, UK William Durante, Columbia, MO, USA Julie Favre, Angers, France Rosa Fernandes, Coimbra, Portugal Hy Fu, Suita, Japan

Junichi Fujii, Yamagata, Japan Noriko Fujiwara, Nishinomiya, Japan Augusto Gallino, Bellinzona, Switzerland Linggen Gao, Beijing, China Janine Gils, Leiden, The Netherlands Louise Glover, Aurora, CO, USA Maik Gollasch, Berlin, Germany Geshon Golomb, Jerusalem, Israel Daniel Gonzalez, Talca, Chile Naoko Goto-Inoue, Tokyo, Japan David Goukassian, Philadelphia, PA, USA Joan Greve, Ann Arbor, MI, USA Rocio Guzmán Ruiz, Córdoba, Spain Tomoki Hashimoto, San Francisco, CA, USA

Anthony Heagerty, Manchester, UK Daniel Henrion, Angers, France Michael Hristov, Munich, Germany Chiang-Ching Huang, Brookfield, WI, USA

Zhiwei Huang, Beijing, China

Yosihiko Ikeda, Suita, Japan

Can Ince, Amsterdam, The Netherlands

Brant Isakson, Charlottesville, VA, USA

Kiyotake Ishikawa, New York, NY, USA Andrei I. Ivanov, Richmond, VA, USA

Daiki Iwami, Sapporo, Japan

Kyeung Min Joo, Suwon, Republic of Korea

Dave Jui, New Haven, CT, USA

Natalia Kasica, Olsztyn, Poland

Masato Katsuyama, Kyoto, Japan

Richard Keijzer, Winnipeg, MB, Canada

Simon Kennedy, Glasgow, UK

Akos Koller, Budapest, Hungary

Petri Kovanen, Helsinki, Finland Thomas Krieg, Cambridge, UK Thomas Kubin, Bad Nauheim, Germany Helena Kuivaniemi, Tygerberg, South Africa
Shant Kumar, Manchester, UK Akhilesh Kumar, Madison, WI, USA

Lih Kuo, Temple, TX, USA

Anton Kutikhin, Kemerovo, Russian Federation Vincenzo La Mura, Milan, Italy Stephan Lange, La Jolla, CA, USA Frédéric Lesage, Montreal, QC, Canada Ding Li, Birmingham, AL, USA Yao Li, Pittsburgh, PA, USA Shan Liao, Calgary, AB, Canada Hui Liu, San Francisco, CA, USA Conglin Liu, Boston, MA, USA Gerald Lutty, Baltimore, MD, USA Zhongwei Lv, Shanghai, China Paolo Madeddu, Bristol, UK Lars Maegdefessel, Munich, Germany Sigrid Maerz, Münster, Germany Luis A. Martinez-Lemus, Columbia, MO, USA

John McGuire, St. John's, NL, Canada Seung-Kee Min, Seoul, Republic of Korea Tohru Minamino, Niigata, Japan Lucia Morbidelli, Siena, Italy Sandrine Morel, Geneva, Switzerland Ryuichi Morishita, Osaka, Japan Alan R. Morrison, Providence, RI, USA Roberto Motterlini, Créteil, France Amie Moyes, London, UK Michael J. Mulvany, Aarhus, Denmark Isamu Murata, Sakado, Japan Tim Murphy, Sydney, NSW, Australia Eduardo Nava, Albacete, Spain Stuart A. Nicklin, Glasgow, UK Liming Nie, Xiamen, China Holger Nilsson, Göteborg, Sweden Graeme F. Nixon, Aberdeen, UK Jerzy-Roch Nofer, Münster, Germany Ryszard Nosalski, Glasgow, UK Alexander Oberhuber, Düsseldorf, Germany Takeshi Otsuki, Ryugasaki, Japan Gary K. Owens, Charlottesville, VA, USA Feyzan Özdal-Kurt, Manisa, Turkey Yoonjung Park, Houston, TX, USA 
Tianqing Peng, London, ON, Canada

Heloise Anne Pereira,

Oklahoma City, OK, USA

Michael R. Pinsky, Pittsburgh, PA, USA

Joachim Pircher, Munich, Germany

Axel R. Pries, Berlin, Germany

Tan Qiang, Qinhuangdao, China

Yanwen Qin, Beijing, China

Uwe Querfeld, Berlin, Germany

Markus Rehm, Munich, Germany

Mathieu Rémond, Amiens, France

Damiano Rizzoni, Brescia, Italy

Lynn Roth, Antwerp, Belgium

Dorianna Sandona, Padova, Italy

Klaus-Dieter Schlueter, Giessen, Germany

Thomas Schmitz-Rixen,

Frankfurt am Main, Germany

Patrick Schober, Amsterdam,

The Netherlands

Rudolf Schubert, Mannheim, Germany

Mark Segal, Gainesville, FL, USA

Carla Sens-Albert, Heidelberg, Germany

Masa-Aki Shibata, Takatsuki, Japan
Molly Shoichet, Toronto, ON, Canada Christoph Siebenmann, Stockholm, Sweden

Ulf Simonsen, Aarhus, Denmark

Amrit Pal Singh, Amritsar, India

Sergey V. Smirnov, Bath, UK

Martin Snoer, Copenhagen, Denmark

Lydia Sorokin, Münster, Germany

Flavia Souza-Smith, New Orleans, USA

Aneta Stefanovska, Lancaster, UK

Guo-Hua Tang, Shanghai, China

John M. Tarbell, New York, NY, USA

George Tellides, New Haven, CT, USA

Sean Thatcher, Lexington, KY, USA

Christopher Torrens, Southampton, UK

Aaron J. Trask, Columbus, OH, USA

Markel Troy, Indianapolis, IN, USA

Md Ashik Ullah, Brisbane, QLD, Australia

Zoltan Ungvari, Oklahoma City, OK, USA

Victor W. van Hinsbergh, Amsterdam, The Netherlands

Babu Budikote Venkatappa, Kuopio, Finland
Hans Vink, Maastricht, The Netherlands Agostino Virdis, Pisa, Italy Ysander von Boxberg, Paris, France Dick Wågsäter, Linköping, Sweden Qinghua Wang, Toronto, ON, Canada Wei Wang, Changsha, China

R. Clinton Webb, Augusta, GA, USA

Erika Wittchen, Chapel Hill, NC, USA

Xianzheng Wu, Shanghai, China

Vishal Yadav, Morgantown, WV, USA

Jianyun Yan, Guangzhou, China

Daping Yang, Harbin, China

Tadashi Yoshida, Tokyo, Japan

Masaaki Zaitsu, Sapporo, Japan

Elisabeth Zeisberg, Göttingen, Germany

Xiaohui (Frank) Zhang, Bethlehem, PA, USA

Nan Zhang, Saint Louis, WA, USA

Min Zhang, Shanghai, China

Guoxing Zheng, Rockford, IL, USA

Ting Zhou, Madison, WI, USA

Thanks to the Reviewers

J Vasc Res 2017;54:386-387 